\title{
Deutschsprachige slowenische Zeitungen und Banus Jelačić im Revolutionsjahr 1848 \\ Eine Analyse am Beispiel von der Cillier Zeitung und dem Sloveniens Blatt
}

\begin{abstract}
Nach der Aufhebung der Zensur im März 1848 erschienen überall in der Habsburger Monarchie neue Zeitungen und die Pressefreiheit fand auch in den Ländern mit slowenischer Bevölkerung ihren konkreten Niederschlag. In diese Recherche wurden ausschließlich die durch die Revolution "geborenen“ deutschen Zeitungen einbezogen. Ihre Sprache war Deutsch, weil die deutsche Sprache in jener Zeit den gebildeten slowenischen Bürgern viel vertrauter war als Slowenisch. Da das Jahr 1848/49 durch die Revolution geprägt war, und die Zeitungen Spiegel ihrer Zeit sind, trugen diese einen ausgeprägt politischen Charakter. In diesem Zusammenhang wollten wir ihre Beziehungen zum Banus Josip Jelačić untersuchen, vor allem die Reaktionen auf seine Aktivitäten im Revolutionsjahr.
\end{abstract}

Schlüsselwörter: Pressefreiheit, Revolution 1848/49, Josip Jelačić, deutschsprachige slowenische Zeitungen

\section{Einleitung}

Obwohl die im Revolutionsjahr herausgebrachten (deutschsprachigen) Zeitungen im Mittelpunkt des vorliegenden Beitrages stehen, sollen zunächst auch die Zeitungen genannt werden, die im Vormärz in den Gebieten mit slowenischer Bevölkerung gelesen wurden. In der ersten Hälfte des 19. Jahrhunderts waren es in diesem Raum vor allem Zeitungen aus Graz oder Triest, die großen Einfluss auf die Gestaltung der öffentlichen Meinung der damaligen gebildeten slowenischen Bevölkerung hatten. In den bürgerlichen Kreisen war die Augsburger Allgemeine Zeitung sehr beliebt, ${ }^{1}$ die aber verboten wurde. So mussten sich eifrige Zeitungsleser dieser Zeit mit den offiziellen Zeitungen zufriedengeben, die nach den Landeshauptstädten benannt wurden, also Laibacher Zeitung, Gratzer Zeitung, Journal des oesterreichischen Lloyd oder Kla-

Marija Mojca Peternel, Abteilung für Germanistik mit Nederlandistik und Skandinavistik, Universität in Ljubljana, Philosophische Fakultät Ljubljana, Aškerčeva 2, 1000 Ljubljana, Slowenien, E-mail: mojca.peternel@ff.uni-lj.si

1 Vgl. Marija Mojca Peternel, „Slovenci v luči augsburškega Allgemeine Zeitung v revolucionarnem letu 1848/1849“, Časopis za zgodovino in narodopisje 87 (2016), Nr. 1/2: 21-41. 
genfurter Zeitung. Illyrisch orientierte Bürger lasen auch gerne Zeitungen aus Zagreb (Agramer Zeitung) oder Prag (Pražské noviny).

In den mit Slowenen besiedelten Gebieten ist derzeitig auch die slowenische Zeitung Lublanske Novize zu erwähnen, die seit dem Jahre 1843 als Kmetijske in rokodelske novice wöchentlich erschien und deren verantwortliche Redakteur den damals hochangesehenen Arzt und Tierarzt Dr. Janez Bleiweis war. ${ }^{2}$

Im Mittelpunkt unserer Recherche sind aber die im Revolutionsjahr "geborenen“ deutschsprachigen Zeitungen in den slowenischen Ländern, die in der slowenischen Geschichtsschreibung lange Zeit übersehen wurden. Der Grund dafür liegt hauptsächlich in der Anwendung der deutschen Sprache. Erst nach der Unabhängigkeit Sloweniens wurde anerkannt, dass vor dem Jahre 1848 die deutsche Sprache die Kulturund Umgangssprache der slowenischen Gebildeten war. Um das zu erklären, soll an der Stelle noch ein wichtiger Aspekt angesprochen werden, der einen großen Einfluss auf das damalige Leben (und somit auch auf die Zeitungen) hatte. Es handelt sich um das berühmte slowenische Nationalprogramm aus dem Jahre 1848, ${ }^{3}$ dessen zweiter Punkt unter anderem die Vereinigung aller Slowenen in Österreich sowie die Einführung der slowenischen Sprache an Schulen und Behörden vorsah. Die Tatsache, dass die nach erlassener Pressefreiheit herausgegebenen Zeitungen im slowenischen Raum ausgerechnet in deutscher Sprache abgefasst waren, mag deswegen zunächst unlogisch erscheinen. Allerdings war vielen klar, dass es eine große Diskrepanz zwischen den prinzipiellen Forderungen (im Nationalprogramm) und den realen Möglichkeiten gab. Der so formulierte zweite Punkt des slowenischen Nationalprogramms ging unter anderem davon aus, dass Deutsch die Kommunikationssprache sowohl der damaligen Intellektuellen als auch der Slawen der Habsburger Monarchie (auch auBerhalb der Monarchie) war, und die Slowenen bildeten keine Ausnahme. Die slowenischen Intellektuellen kamen in Kontakt mit zeitgemäßen politischen Strömungen während ihres Studiums in Wien oder Graz. So benutzte sogar der größte slowenische Dichter France Prešeren, ${ }^{4}$ der auch in Wien studierte, in seinen Gesprächen (nicht nur) mit Anton Auersperg - Anastasius Grün ${ }^{5}$ lieber Deutsch.

Auch slowenische Beamte drängten nicht auf eine sofortige Einführung des Slowenischen als Amtssprache. Sie waren oft nicht in der Lage, die slowenische Sprache im öffentlichen oder sogar in ihrem privaten Leben uneingeschränkt zu verwenden.

2 Bleiweis Ritter Trsteniški, Demeter (3. April 1871 Ljubljana/Laibach - †10. Januar 1928 Ljubljana/ Laibach), https://www.slovenska-biografija.si (Zugriff 22. 7. 2019).

3 Vgl. Stane Granda, Prva odločitev Slovencev za Slovenijo (Ljubljana: Korenine, 1999).

4 France Prešern (3. 12. 1800, Vrba/Velden - †8. 2. 1849, Kranj/Krainburg), https://www.slovenska-biografija.si (Zugriff 22. 7. 2019).

5 Anton Auersperg (11. 4. 1806, Ljubljana/Laibach - †12. 9. 1876, Graz), https://www.slovenska-biografija.si. Vgl. Mira Miladinović Zalaznik, Stane Granda (Hg.): Anton Aleksander Graf Auersperg - Anastazij Grün. Razprave [Anton Alexander Graf Auersperg Anastasius Grün. Abhandlungen] (Ljubljana: Nova revija, 2009). 
Josip Jelačić, ${ }^{6}$ als der Hauptprotagonist der kroatischen Nationalbewegung und der Revolution von 1848/49, in der er gegen die ungarischen Revolutionäre militärisch vorging und dessen Einsatz für den Erhalt der Habsburger Monarchie ihm den Namen des „Retters Österreichs“ einbrachte, gilt er als eine der bedeutendsten Persönlichkeiten der kroatischen Geschichte des 19. Jahrhunderts. ${ }^{7}$ Als solche war er auch den damaligen Slowenen (und somit auch den Zeitungslesern) bestimmt nicht unbekannt. In der slowenischen Geschichtsschreibung ist bisher über ihn zwar noch keine Monografie erschienen, jedoch kommt er in allen Lehrwerken für Geschichte vor. Neben Ljudevit Gaj ${ }^{8}$ ist er bestimmt im slowenischen Raum die bekannteste Persönlichkeit aus der kroatischen Geschichte überhaupt.

\section{Der Zeitungsboom im Jahre 1848}

Am Tag nach dem Ausbruch der Revolution 1848 in Wien, als Kaiser Ferdinand I. (1793 -1875) unter anderem die Aufhebung der Zensur und die Pressefreiheit erklärte, war die Begeisterung in der Residenzstadt unermesslich. Schon in den Märztagen wurden zahlreiche Zeitungen überall in der Monarchie herausgebracht, die an den Straßenecken aller Großstädten angeboten wurden: „An allen Ecken häufen sich Plakate an allen öffentlichen Plätzen, in allen Gassen erscholl der Ruf von Colporteurs und frechen Weibern, welche diese Producte der zügelassesten Preßfreiheit ausriesen und unter allen Klassen der Bevölkerung verbreiteten, der beste Weg die Gesinnung der charakterlosen menge allmälig zu vergiften. ${ }^{\text {"9 }}$ Ähnliches wie in Wien spielte sich auch in Graz ab: „An den Häusern der Gässen, an den Ecken der Straßen kleben Plakate und Ankündigungen, eine über der andern, Aufrufe und Anordnungen, Warnungen und Ankündigungen kommen an ihnen zum Vorscheine und verschwinden wieder und lösen sich oft mehrmals im Tage, in den Buchläden und Zeitungscomptoirs, vor der Post und Sparkasse drängt sich eine Menge von Menschen wartend, kaufend, lesend alle zahlreichen Flugschriften, Extrablätter, Zeitungen, welche begierig gekauft und noch begierig verschlingen werden." ${ }^{10}$

Der Zeitungsboom bedeutete aber nicht unbedingt qualitativ hochwertige Zeitungen, und auch die Provisorische Verordnung gegen den Mißbrauch der Presse vom 18. Mai 1848, die Druckern und Redakteuren mit hohen Strafen drohte, konnte diese

6 Josip Jelačić (16. 10. 1801, Petrovaradin/Peterwardein - †20. 5. 1859, Zagreb/Agram), http://hbl. lzmk.hr (Zugriff 22. 7. 2019). Vgl. Kristina Milković, Josip Jelačić 1830.-1848. Plemić, časnik $i$ preporoditelj u društvima „staroga režima“ (Magisterarbeit, Sveučilište u Zagrebu, 2009), 66-67. Milković, Josip Jelačić 1830.-1848., 296.

8 Ljudevit Gaj (8. 7. 1809 Krapina/Grabing - †20. 4. 1872, Zagreb/Agram), http://hbl.lzmk.hr (Zugriff 22. 7. 2019).

$9 \quad$ Hermann Meynert, Geschichte der Ereignisse in der österreichischen Monarchie während der Jahre 1848 u. 1849 in ihren Ursachen und Folgen (Zugleich als Suppl. zu „Gesch. Oesterreichs“ vom Verfasser) (Wien: Verlag und Druck von Carl Gerold und Sohn, 1853), 284.

10 Ferdinand Gatti, Die Ereignisse des Jahres 1848 in der Steiermark (Graz: In Commision bei J. Kienrich, 1850), 44. 
Tatsache nur zu einem bestimmten Grad mildern. ${ }^{11}$ Laut der erwähnten Verordnung wurden unter anderem unwahre oder höhnische Darstellungen des österreichischen Kaiserstaates, Verletzungen der Sittlichkeit, Gotteslästerung usw. mit schwerer Kerker- und/oder Geldstrafe geahndet. ${ }^{12}$ Dem Zeitungswesen wurden auch durch den $\$ 4$ der am 23. Oktober herausgebrachten Proklamation die Flügel gestützt: „Auf die Dauer des Belagerungszustandes sind alle Zeitungsblätter zu suspendiren, mit Ausnahme der Wiener Zeitung, welche sich bloß auf officielle Mittheilungen zu beschränken hat. ${ }^{\text {"13 }}$ Das geschah nachdem mit militärischer Gewalt die Oktoberrevolution in Wien niedergeschlagen worden war.

Die neuen Zeitungen unterschieden sich nicht nur inhaltlich, sondern auch durch die Häufigkeit der Ausgaben. Einige erschienen sogar nur einmalig, wofür es mehrere Gründe gab. Unter anderem sind der immer schwächer werdende Revolutionsgeist und die knappen finanziellen Mittel zu erwähnen. Das Zeitungsleben entfachte sich damals oft im Geist des Dilettantismus und die Zeitungen erschienen hauptsächlich, weil man ein starkes Interesse an ständig wechselnden Ereignissen hatte. Es gab keine im heutigen Sinne professionelle Journalisten und so waren gedruckte Berichte und Zeitungsnotizen häufig einseitig und subjektiv, weil sie von unterschiedlichen Informationsvermittlern geschrieben wurden. Oft mangelte es gerade aus diesem Grund an kritischen Betrachtungen und Objektivität.

Das Lesepublikum der neuen (,,jungen“) Zeitungen war genauso unterschiedlich. Es handelte sich demnach um ganz spezifische Zeitungen (z. B. Der Humorist) oder um solche, die unterhalten oder belehren wollten (z. B. Allgemeine Theaterzeitung).

Langsamer als in den Großstädten verlief das Zeitungsleben in den Kleinstädten. In den mit Slowenen besiedelten Ländern fand die Pressefreiheit relativ früh ihren konkreten Niederschlag. Anfangs wurden nur Flugblätter, später auch Zeitungen herausgegeben. ${ }^{14}$ Zuerst erschien in Celje (Cilli) das Cillier Wochenblatt (1. April 1848), das nach kurzer Zeit (4. Juli 1848) in Cillier Zeitung ${ }^{15}$ umbenannt wurde. Dieser folgte in Novo Mesto (Neustadt/Rudolfswert) die Zeitung Sloveniens Blatt (10. Mai 1848). ${ }^{16}$ Der Bedarf an politischen Mitteilungen und Informationen war sehr groß, und so erschien auch bei der „alten“ Laibacher Zeitung in Ljubljana (Laibach) am 20. Juli 1848 die erste Nummer des Politischen Blattes als Extra-Beilage zur Laibacher Zeitung. Im

11 Provisorische Verordnung gegen den Mißbrauch der Presse, Anhang zur Laibacher Zeitung, Laibacher Zeitung (Ljubljana/Laibach), 23. 5. 1848, unpaginiert; Provisorische Verordnung gegen den Mißbrauch der Presse (Ljubljana/Laibach) Besondere Beilage zu Laibacher Zeitung, 18. 7. 1848, unpaginiert; und Meynert, Geschichte der Ereignisse in der österreichischen Monarchie während der Jahre 1848 u. 1849 in ihren Ursachen und Folgen, 281.

12 Provisorische Verordnung gegen den Mißbrauch der Presse, Anhang zur Laibacher Zeitung, Laibacher Zeitung (Ljubljana/Laibach), 23. 5. 1848, unpaginiert, \$7.-\$.22.

13 Ebd., 10.

14 Vgl. Stane Granda, „Sloveniens Blatt - časopis iz leta 1848“, Zgodovinski časopis 48 (1994), Nr. 2: 231.

15 Vgl. Marija Mojca Peternel, Cillier Wochenblatt (Zeitung): celjski nemški časopis iz leta 1848 (Celje: Zgodovinsko društvo, 2006).

16 Vgl. Granda, „Sloveniens Blatt - časopis iz leta 1848“, 231-252. 
Sommer begann auch die Kirche, auf Anregung des lokalen Bischofs Janez Zlatoust Pogačar, ${ }^{17}$ die slowenische Slovenski cerkveni časopis und die deutschsprachige Laibacher Kirchenzeitung, die am 6. Juli 1848 zum ersten Mal erschien, herauszugeben. ${ }^{18}$ Neben den erwähnten Zeitungen erschienen in den Ländern mit slowenischer Bevölkerung auch neue slowenische Zeitungen: in Ljubljana die erste politische Zeitung Slovenija in slowenischer Sprache, in Celje die Celjske Slovenske Novine (Cillier Slowenische Zeitung), und dazu noch weitere, wie die erste slowenische Zeitung Vedež in Triest.

Die verkündete Pressefreiheit wurde jedoch auch mit anderen Aufgaben des Zeitungswesens verbunden. Während die Zeitungen des Vormärz dem Lesepublikum vor allem Unterhaltung brachten, mussten die „neuen“ Zeitungen aktuelle, vor allem politische Ereignisse - denn das Revolutionsjahr 1848/1849 war ja ein politisches Jahr - auf eine möglichst einfache Weise erklären und die Leser belehren. Damit die Presse diese neue Aufgabe erfüllt, sollten ihr „die Männer von Talent und Wissen zu Hilfe" kommen. ${ }^{19}$

Dass das Revolutionsjahr 1848/49 ein politisches Jahr war und dass sich die Tatsache in dem damaligen Zeitungswesen widerspiegelte, zeigt unsere Recherche der ,jungen“ Zeitungen, die nach dem Ausbruch der Revolution im damaligen slowenischen Raum herausgegeben wurden. Sie behandelten aktuelle Themen und somit auch die wichtigsten Persönlichkeiten des Jahres, wie z.B. den Banus Josip Jelačić. Unter den „jungen“ deutschsprachigen Zeitungen gab es Berichte über ihn nur in der Cillier Zeitung und im Sloveniens Blatt. Das Extrablatt zur Laibacher Zeitung fokussierte sich auf andere brennende Themen. In der Laibacher Kirchenzeitung dagegen erschienen Beiträge über aktuelle kirchliche und gesellschaftliche Fragen, deren Autoren aber auch aus den kroatischen Gebieten stammten. Aus der kroatischen Küstenregion befasste sich Leo Wonzhina ${ }^{20}$ in einem langen Artikel mit der damals wichtigen Frage des Zölibats. ${ }^{21}$ Der Grund, warum die Laibacher Kirchenzeitung so zurückhaltend gegenüber Kroatien war, lag vermutlich auch in den Bestrebungen einiger Kroaten, die römische Kirche mit der griechisch-orthodoxen zu vereinigen. ${ }^{22}$

17 Janez Zlatoust Pogačar (22. 1. 1811, Vrba/Velden - †25. 1. 1884, Ljubljana/Laibach), https://www.slovenska-biografija.si (Zugriff 22. 7. 2019). Vgl. Matjaž Ambrožič, Ljubljanski knezoškof dr. Janez Zlatoust Pogačar (Ljubljana: Acta Ecclesiastica Sloveniae, 2000).

18 Marija Mojca Peternel, „Die Laibacher Kirchenzeitung: Pressefreiheit und Kirche im Revolutionsjahr 1848“, Blätter für Heimatkunde 89 (2015), Heft 1/2: 25-41.

19 Del Cott, „Die Journalistik, Laibacher Zeitung“ (Ljubljana/Laibach), 24. 6. 1848, 450.

20 Leon Stanislav Vončina (7. 4. 1826 chroatische Küstenregion (Novi Vinodolski) - †4. 11. 1874 Ljubljana/Laibach), https://www.slovenska-biografija.si (Zugriff 22. 7. 2019).

${ }^{21}$ Leo Wohnzhina, „Der Cölibat und die katholische Kirche mit besonderer Rücksicht auf die anticölibatischen Bewegung in Croatien“, Laibacher Kirchenzeitung (Ljubljana/Laibach), 24. 8. 1848, 58-59, 7. 10. 1848, 75-77, 14. 9. 1848, 81-83, 21. 9. 1848, 89-91, 26. 10. 1848, 128-131, 2. 11. 1848, 137-139.

22 Josip Neustädter, Ban Jelačić i događaji u Hrvatskoj, od godine 1848 (Zagreb: Školska knjiga,1998), Band 1, 323. 
Der kroatische Banus war ziemlich beliebt im slowenischen (politischen) Raum, denn im Gegensatz zu den Kroaten hatten sie keine Persönlichkeit auf so hoher politischer Position. Die Slowenen hatten keine große staatliche Tradition und fast keine national denkende Aristokratie ${ }^{23}$ oder einen hochrangigen slowenischen Vertreter im damaligen politischen Leben. Und der Banus war gerade das, was sie vermissten, ein Amtsträger mit hohem militärischem Dienstgrad, dessen nationale Orientierung klar war. Manche sahen in ihm eine vertrauenswürdige Person und den Vertreter aller Kroaten.

Wien war ein traditioneller Treffpunkt der slowenischen und kroatischen Intellektuellen in der Habsburger Monarchie, die sich vor, aber auch während der Märzrevolution begegneten. Da entstand auch das erste Konzept der angehenden politischen Verbindung der Nationen in der Habsburger Monarchie und dabei unterstützten die slowenischen Vertreter den kroatischen Kampf gegen das ungarische Joch. Auch im schon erwähnten Nationalprogramm wurde eine engere Verbindung mit den Kroaten vorgesehen und diese Zuneigung war bestimmt eine Folge der Illyrischen Bewegung. ${ }^{24}$ Nach den Oktoberereignissen jedoch, als Jelačić half, den Aufstand in Wien niederzuschlagen, war er bei den slowenischen Liberalen, die die Revolution unterstützten, nicht mehr so beliebt.

\section{Die Cillier Zeitung und Josip Jelačić}

Das Verhältnis der Cillier Zeitung zu dem Banus begann sich erst nach dem Sommer 1848 richtig zu gestalten. Generell brachten die ersten Zeitungsausgaben sehr selten Nachrichten aus den Ostgebieten. Das erste Aufsehen erregende Nachricht aus diesen Gebieten war ein Text über den Besuch der kroatischen Delegation bei dem Cillier Društvo za pomirjanje obeh narodnosti (Verein zur Vermittlung der deutschen und slawischen Interessen), ${ }^{25}$ veröffentlicht Ende Mai. In der Stadt lebten neben den Slowenen auch Deutsche, und die Nationalkonflikte traten im Jahr 1848/1849 immer deutlicher zu Tage. Die wichtigste Aufgabe des o.g. Vereins (schon dem Namen nach) war, die beiden in der Stadt lebenden Nationen zu versöhnen. Dabei wäre auch eine engere Verbindung mit den Kroaten denkbar gewesen. Da die Cillier Zeitung als die Sprecherin des Vereins galt, war auch ihre erste Aufgabe die Verständigung und Toleranz zwischen den Nationen zu unterstützen. Darum scheint es logisch, dass die Delegierten aus Kroatien, nach den Worten eines unbenannten Autors, in der Stadt mit Begeisterung empfangen wurden. ${ }^{26}$ Außer einer Begeisterung kann man der Nachricht nichts Näheres über die Delegation entnehmen. Es handelte sich dabei bestimmt nicht um die kroatische Delegation mit dem Banus, die auf der Durchreise

23 Andrej Rahten, Zavezništva in delitve. Razvoj slovensko-hrvaških političnih odnosov v Habsburški monarhiji (Ljubljana: Nova revija, 2005), 17.

24 Rahten, Zavezništva in delitve, 35-37. Siehe auch Fran Ilešič, O slovensko-hrvatski zajednici 1848/49, Bleiweisov zbornik (Ljubljana: Matica Slovenska, 1909), 279-280.

25 Wochenschau, Cillier Wochenblatt, 25. 5. 1848, 42.

26 Wochenschau, Cillier Wochenblatt, 47. 
zum Kaiser nach Innsbruck war, da es erst im Juni passierte. ${ }^{27}$ Und die Nachricht ist bestimmt auch nicht mit seiner vorherigen Reise nach Wien zu verbinden, wo er den Eid als Banus am 8. April ablegte und zum Feldmarschallleutnant und Befehlshaber der kroatischen Militärgrenze ernannt wurde. ${ }^{28}$

Der erwähnte Cillier Verein unterstützte den Kaiser und die Idee von einem starken Österreich und war somit auch für die Verbindung mit den Kroaten. Deshalb war die Cillier Zeitung Josip Jelačić zunächst gut gesonnen. Das beweist der im August gedruckte Bericht über seinen Besuch in der Stadt Celje, in der er sich mit dem Abgeordneten Vincenc Gurnik traf. Dieser war von der Präsenz des Banus völlig begeistert: „Ich hatte die Ehre dem Banus abgesondert meine Aufwartung zu machen, und ihn im Namen der Slovenen der höchsten Verehrung, und der wärmsten Liebe, sein Volk, das treue und tapfere Volk der Croaten aber durch ihn unserer innigsten Sympathien zu versichern. [...] Meine Brust war so voll der Begeisterung als ich dem ritterlichen, und doch so herzlichen gemüthreichen Banus gegenüber stand, daß ich für meine Worte kein Gedächtniß behielt. Daß er mich aber verstand, bewies mir sein so herzliches, liebevolles Begegnen. “29

Der Abgeordnete versprach dem Banus Unterstützung und lobte seinen Kampf für den Kaiser: „Als ich ihm sagte, daß wir Südslaven auf ihn als den Mann sehen, der als Österreichs Grundpfeiler die constitutionelle Monarchie zu schützen berufen ist, daß wir zu diesem Ende uns um ihn zu scharren bereit sind, da fiel mir der Banus, sichtlich ergriffen, ins Wort und sagte: ,Ich verdiene so viel Auszeichnung nicht, ich bin kein außergewöhnlicher Mensch, ich bin ein schlichter aber ehrlicher Mann, glühe für die Freiheit, meinen Kaiser und mein Volk. Dank Ihnen und Ihren slovenischen Brüdern, die mir als stammverwandte Brüder so theuer sind. ““30

Warum die Zeitung bis Oktober trotz dieser Begeisterung keine Notizen über den Banus mehr brachte, ist nicht ganz klar. Ihre Bestrebungen, die möglichst aktuellen Nachrichten zu drucken, könnte ein Grund dafür sein. Die Zeitung befasste sich nämlich sehr umfangreich mit dem Prager Kongress, der im Sommer stattfand.

Erst nach dem Zusammenbruch der Oktoberrevolution von 1848, als der Revolutionsgeist deutlich nachließ (das auch mit strengeren Presseverordnungen im Zusammenhang stand), wurden die Berichte über Jelačić häufiger. Ab diesem Zeitpunkt kann in der Zeitung eine andere (und auch endgültige) Beziehung zu dem Banus festgestellt werden.

27 Stephan Pejaković, Aktenstücke zur Geschichte des kroatisch-slavonischen Landtages und der nationalen Bewegung vom Jahre 1848: mit einem Anhange, enthaltend: Die wichtigsten LandtagsAkten vom Jahre 1861, und das Programm von O. Ostrožinski aus dem Jahre 1848: Nebst Porträt des Ban Jellačić (Wien: Mechitharisten-Buchdruckerei, 1861), 100.

28 Jaroslav Šidak, Studije iz hrvatske povijesti za revolucije 1848-49 (Zagreb: Centar za povijesne znanosti Sveučilišta, Odjel za hrvatsku povijest, 1979), 49-52; und Nikša Stančić, „JELAČIĆ, Josip (Jellačić, Jellachich; Joseph)“, in: Hrvatski biografski leksikon, Band 6 (Zagreb: Leksikografski zavod Miroslav Krleža, 2005), 394-401; Pejaković, Aktenstücke, 100.

29 Cillier Zeitung, 4. 8. 1848, 120.

30 Cillier Zeitung, 4. 8. 1848, 120. 
Die Geschehnisse in Wien, in denen Jelačić bestimmt ein wichtiger Protagonist war, verursachten große Enttäuschung bei einem unbekannten Autor, der für die Cillier Zeitung unter anderem schrieb: „Tief erschüttert von der vernichtenden Gewalt der Ereignisse in der Residenc, wo Bürger gegen Bürger, Waffensbrüder gegen Waffens-

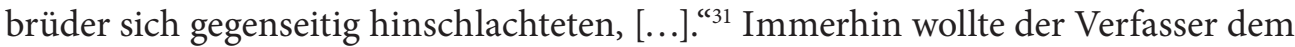
Kaiser treu bleiben und forderte auf: „Aber ein Wort müssen wir mit gewaltiger Stimme allein zurufen: Treue Ergebenheit für den Monarchen und Achtung vor den Beschlüssen des Reichstags, solange derselbe nicht durch die Gewalt der Bajonette eingeschüchtert ist. “32

Die Cillier Zeitung widmete den Wiener Ereignissen im Oktober viel Aufmerksamkeit. Die Berichte aus der Residenzstadt erschienen ausschließlich auf dem Titelblatt, entsprechend ihrer hohen Wichtigkeit. Ob diese Berichte mehrerer Autoren waren, konnte nicht festgestellt werden. Der gleiche Schreibstil weist aber darauf hin, dass es sich um einen einzigen Autor handelt. Bei den erwähnten Ereignissen wurde besonders große Aufmerksamkeit der Tatsache gewidmet, dass die Stadt Celje es abgelehnt hatte, den Revolutionären in Wien Hilfe zu leisten. So die Mitteilung aus der Stadt: „Das Aufgeboth nach Wien, dessen Garde, wie des Finanzministers Depesche lautete, erschöpft, weßhalb die Hilfe der steiermarkischen wünschenwerth sei, hat hier aus Gründen, die wir nächstens besprechen werden, keinen Anklang gefunden. "33 Dass es dem Autor (vielleicht auch dem Redakteur) nicht gleichgültig war, bewies die Fortsetzung in der nächsten Ausgabe: „In unserer Kreisstadt fand die Sache keinen Anklang, und wir gestehen selbst, daß weder die Worte der Depesche welche nur von einem dringenden Wunsche der Bevölkerung von Gratz sprach, noch das Auftreten es Abgeordneten Vertrauen oder Begeisterung erwecken konnten." ${ }^{34}$ Zwischen den Zeilen spürt man die Unzufriedenheit mit den lokalen politischen Protagonisten. Das Verhalten der dortigen Stadtabgeordneten in dieser kritischen Zeit fand der unbenannte Autor unverantwortlich und bezeichnete es als kein gutes Vorbild für das Volk.

Grund für die Vermutung, dass hinter dem unbekannten Autor auch gleichzeitig der liberale Redakteur stecken könnte, liegt in der Veränderung des Zeitungsinhalts nach dem Oktober 1848. Die Enttäuschung mit den erwähnten Ereignissen widerspiegelt sich in dem Inhalt der Zeitung zu einem solchen Maße, dass ab diesem Zeitpunkt fast keine Autorenartikel, sondern fast ausschließlich aus anderen Zeitungen nachgedruckte Artikel, auch ohne Bemerkungen des Redakteurs veröffentlicht wurden.

Dass in diesen stürmischen Zeiten die Cillier Zeitung keine Begeisterung mit der damaligen Reaktion von Jelačić zeigte, beweisen auch die Worte: „Das steiermärkische Aufgeboth ist bei dem Umstande, daß das Vordringen nach Wien durch die Vorposten des Feindes gehindert war, bereits zurückgekehrt. Obschon auf solche Weise der Zweck nicht erreicht wurde, so haben doch diese Männer, worunter sehr zahlreiche

Wien, Cillier Zeitung, 10. 10. 1848, 193.

Wien, Cillier Zeitung, 10. 10. 1848, 193.

Cilli, Cillier Zeitung, 13. 10. 1848, 200.

Cilli, Cillier Zeitung, 17. 10. 1848, 203. 
Garden von Marburg, Windisch Feistritz, Pettau bewiesen, daß sie mit selbstständigen Urteheile die Gefahr der jungen Freiheit erkannt haben und ihr muthig entgegen treten werden, gleichviel von welcher Seite sie drohen mag. Sie haben es erkannt, daß der Ban unberechtigt sich in unsere Angelegenheiten mit bewaffneten Hand mischte, daß es sich hier nicht um die Nationalität, sondern um Aufrechterhaltung der kaum errungenen Freiheit handle, daß es keinem gleichgiltig sein könne, ob es die Verfassung durch Militärgewalt nach eigenem Gutdünken diktirt wird, oder durch die Berathung unserer Vertreter am Reichstage." ${ }^{\text {"35 }}$

Es sollte hier vielleicht daran erinnert werden, dass der Banus in diesen blutigen Ereignissen nicht eigenhändig handelte. Nachdem das ungarische Parlament aufgelöst worden war und nachdem die kaiserlichen Truppen im Oktober gegen das aufständische Ungarn ziehen wollten, versuchten die Studenten, Arbeiter und aufständische Truppen in Wien das zu verhindern. Ihnen schlossen sich noch Teile der Nationalgarde und der Akademischer Legion an. Es kam zu heftigen Straßenkämpfen, worauf der kaiserliche Hof die Residenzstadt verließ und nach Olmütz floh. Jelačić kam zur Hilfe der kaiserlichen Truppen nach Wien und wurde zum kaiserlichen Kommissar in Ungarn. Diese Oktoberrevolution betrachteten die Kroaten und die Slowenen als eine Drohung für ihre weitere nationale und politische Entwicklung, ${ }^{36}$ das sich auch in der Cillier Zeitung widerspiegelte, denn ihre inhaltliche Änderung nach den Oktoberereignissen ist erkennbar. Dahinter könnte es sich um einen stillen Protest des damaligen liberalen Redakteurs der Zeitung, Vincenc Prasch - Professor am dortigen Gymnasium - gegen das Verhalten der lokalen Politiker bezüglich der Ereignisse handeln.

Die zitierten Ausschnitte zeigen deutlich die veränderte Stimmung in der Beziehung zum Banus. Wenn die Cillier Zeitung am Anfang noch mit Begeisterung über Jelačić berichtete, änderte sich dies nach dem Oktober deutlich. Die Zeitung wollte der Presse- und Meinungsfreiheit weiterhin treu bleiben und brachte über den Banus immer noch Beiträge, jedoch ohne persönliche Note. Obwohl der offene Brief des Abgeordneten Dr. Smrekar ohne Kommentare veröffentlicht wurde, kann man zwischen den Zeilen jedoch ein positives Bild über Jelačić herauslesen: „[...] daß ich der Fahne unseres nachbarlichen, ritterlichen Stammverwandten Banus Jelačić, zur Aufrechthaltung des Kaiserreiches mit Wort und That folge [....]. ${ }^{\text {"37 }}$ Das Interesse an Jelačić zeigte die Cillier Zeitung wenn es um seinen Krieg gegen Ungarn ging, ${ }^{38}$ da sie positiv (sogar begeistert) über seine Aktivitäten aus den Kriegsgebieten berichtete. ${ }^{39}$

35 Cilli, Cillier Zeitung, 17. 10. 1848, 203.

36 Nataša Pogoršek, Slovenska hrvaška vzajemnost (Maribor: Zgodovinsko društvo dr. Franca Kovačiča v Mariboru, 2009), 28. Vgl. auch Petar Korunić, Jugoslavenska ideologija u hrvatskoj i slovenskoj politici: Hrvatsko-slovenski politički odnosi 1848-1870 (Zagreb: Globus: Centar za povijesne znanosti Sveučilišta u Zagrebu, Odjel za hrvatsku povijest, 1986), 114-120.

37 Offenes Schreiben, Cillier Zeitung, 24. 11. 1848, 246.

38 Vgl. Josip Neustädter, Ban Jelačić i događaji u Hrvatskoj, od godine 1848, Band I, 81-117; und Rudolf Horvat, Ban Jelačić. Hrvatski pokret 1848 (Zagreb: Colorprint Zagreb, 1990), Band 2.

39 Cillier Zeitung, 21. 11. 1848, 243. 
Beweise für das fortwährende Interesse an dem Banus zeigen auch andere, jedoch immer mehr im telegrafischen Stil geschriebene Nachrichten. So brachte die Zeitung schon fünf Tage nach der Ernennung des Banus zum Gouverneur von Dalmatien darüber eine kurze Mitteilung. ${ }^{40}$ Danach wurde ohne Kommentar auch eine Mitteilungüber die Verleihung des Leopoldordens an den Ban Ende November veröffentlich. ${ }^{41}$

Das war auch die letzte Nachricht über den Banus in der Cillier Zeitung. Diese Übersicht der Nachrichten zeigt ein deutliches Interesse und auch eine anfängliche Zuneigung. Dies änderte sich jedoch mit den Wiener Ereignissen im Oktober, als die Revolution mit Gewalt niedergeschlagen wurde. Es war auch wahrscheinlich der Grund für die Enttäuschung des (liberalen) Redakteurs der Cillier Zeitung. Außerdem wurde er durch den immer schwächer werdenden Revolutionsgeist tief getroffen. Trotz allem wollte er bis zum Niedergang der Zeitung ${ }^{42}$ dem Prinzip der Aktualität treu bleiben und in diesem Sinne wurden die Nachrichten über den Banus weiterhin gedruckt. ${ }^{43}$

\section{Sloveniens Blatt und Josip Jelačić}

Auch in Novo Mesto (Neustadt) erschien bald nach dem Ausbruch der Revolution ein neues Blatt, Sloveniens Blatt. Die Verhältnisse, unter denen diese Zeitung herausgegeben wurde, unterschieden sich deutlich von denen in Ljubljana oder Celje. Die Stadt Novo Mesto war viel kleiner, mit nur 3.000 Einwohnern. Es waren vorwiegend Händler und Handwerker, die fast alle auch in der Landwirtschaft tätig waren. ${ }^{44}$ In der Stadt lebten 60 Beamte, die ausschließlich Deutsch sprachen, ansonsten waren die Bürger slowenischer Herkunft und mussten Deutsch erlernen. Was die Sprache angeht, waren die Verhältnisse ähnlich wie in Celje, wo die Bürger auch Deutsch sprachen. Den Unterschied merkt man aber bei ihren nationalen Interessen, denn in Novo Mesto waren alle slowenisch orientiert, in Celje nicht. ${ }^{45}$

Das Verhältnis zu dem Banus im Sloveniens Blatt wurde deutlicher erst nach den Ereignissen im Oktober, ähnlich wie in der Cillier Zeitung. Die Geschehnisse in Wien bewertete die Zeitung eher negativ, hatte jedoch Sympathien für die beiden Hauptfiguren der Oktoberereignisse, Radetzky und Jelačić. General Radetzky war bei der hiesigen Bevölkerung sehr beliebt, weil er der regionalen Nationalgarde 200 Gewehre

40 Wien, Cillier Zeitung, 7. 12. 1848, 262. „Nach dem heutigen Reichstags-Blatte ist der Banus von Croatien, Freiherr Josef Jelačić: unter Beibehaltung seiner bisherigen Stellung zum Civil- und Militär Gouverneur von Dalmatien und Fiume ernannt worden.“

41 Wien, Cillier Zeitung, 28. 11. 1848, 250.

42 Die letzte Ausgabe erschien 15. 12. 1848.

43 Wien, Cillier Zeitung, 17. 11. 1848, 239, Cillier Zeitung, 21. 11. 1848, 243, Wien, Cillier Zeitung, 28. 11. 1848, 250, Kremsier, Cillier Zeitung, 1. 12. 1848, 255, Wien, Cillier Zeitung, 7. 12. 1848, 262.

44 Miloš Jakopec, „Časnikarstvo v Novem mestu: pregled od prvih začetkov do II. svetovne vojne.“ Rast 2 (1990): 110-119.

45 Vgl. Stane Granda, Dolenjska v revolucionarnem letu 1848 (Novo mesto: Dolenjska založba, Znanstveno-raziskovalni center, 1995). 
schenkte. ${ }^{46}$ Auch für den Banus pflegte das Sloveniens Blatt große Sympathie, geriet aber bei seinem Verhalten in der Oktoberrevolution in Wien in Verlegenheit. ${ }^{47}$

Über Jelačić berichtete Mitte Oktober ein unbekannter Autor im zweiten Teil seines Artikels Einfluß des südslawischen Krieges auf den österreichischen Gesamtstaat. ${ }^{48}$ Die Rolle des Banus entschuldigte er mit den folgenden Worten: „[...] der Banus will etwas zusammenfügen, was zusammen gehört [...]. ${ }^{\text {“99 }}$ Der Artikel erschien fast genau ein Monat nachdem die kroatischen Truppen unter der Führung von Jelačić in der Nähe von Varaždin die Drau überquert hatten (11. September) und fast zwei Wochen vor der endgültigen Niederlage der Revolution in Wien (31. Oktober). ${ }^{50}$ Die Ereignisse fanden ein großes Echo in der Monarchie und so konnte auch die Provinzstadt Novo Mesto davon nicht unberührt bleiben. Der Redakteur zeigte Interesse an dieser Entwicklung und veröffentlichte noch einen weiteren zweiteiligen Artikel unter dem Titel Die Vollmacht an F. M. Windischgrätz, ${ }^{51}$ in dem er auch einiges über den Banus schrieb. Der Text stammt zwar von einem unbenannten Autor, es könnte sich aber dabei um denselben handeln wie beim oben erwähnten Artikel, denn der Ton und nicht zuletzt der Schreibstil sind ähnlich. Der Verfasser versuchte die Gewaltanwendung des Fürsten nicht nur zu erklären, sondern sogar zu rechtfertigen. Um dies noch deutlicher zu machen, wurde das Ganze noch optisch hervorgehoben: „[...]; ja, er sei anwendbar, wenn das, was realisiren soll, die Beseitigung eines Uebels ist, und das Uebel durch welches solche Beseitigung geschieht ein geringeres ist, als das zu beseitigende. “52

Der Autor weiß, dass der Staat in einer Krise sei, aus der nur zwei Wege führen könnten: einer in den Untergang und der andere in eine bessere Zukunft. Er sei zwar gegen Gewalt, wisse jedoch, dass das jetzige Übel geringer sei als das frühere. Im zweiten Teil des Artikels stellt er den Banus sehr positiv dar: „[...] es war das eines Mannes der aus der Mitte der europäischen Civilisation entsprossen neben tiefer Consequenz und militärischer Strenge auch die Freiheit liebt; [...]. ${ }^{{ }^{53}}$ Er meint, sein Verhalten sei sehr geeignet und kultiviert, also ganz das Gegenteil von den sonst klischeehaften Bildern über die Kroaten. ${ }^{54}$

\footnotetext{
Locales, Sloveniens Blatt, 17. 10. 1848, 64.

47 Granda, „Sloveniens Blatt - časopis iz leta 1848“, 240.

48 Einfluß des südslawischen Krieges auf den österreichischen Gesamtstaat, Sloveniens Blatt, 10. 10. 1848, 57-58 und 17. 10. 1848, 61-62.

49 Einfluß des südslawischen Krieges auf den österreichischen Gesamtstaat, Sloveniens Blatt, 17. 10. $1848,61-62$.

50 Vgl. Rudolf Horvat, Ban Jelačić. Hrvatski pokret 1848, 258-381.

51 Die Vollmacht an F. M. Windischgrätz, Sloveniens Blatt, 31. 10. 1848, 70-71 und 7. 11. 1848, 7-75.

52 Die Vollmacht an F. M. Windischgrätz, Sloveniens Blatt, 31. 10. 1848, 70.

53 Die Vollmacht an F. M. Windischgrätz, Sloveniens Blatt, 7. 11. 1848, 74.

54 „An der Stelle sollte noch einmal an seine Fähigkeiten erinnert werden. Jelačić beherrschte viele Sprachen (Deutsch, Französisch, Magyarisch, Italienisch und Latein). Er war ehrlich, edel und gutmutig, froh, einfach, freundlich, eher sanft als streng. Bei der Arbeit war er gewandt und sehr fähig." Rudolf Horvat, Ban Jelačić. Prvi svezak, Hrvatski pokret 1848, 5.
} 
Über Jelačić schrieb ein unbekannter Autor (vielleicht wieder der gleiche) im Artikel Einflu $\beta$ des südslavischen Krieges auf den österreichischen Gesammtstaat, ${ }^{55}$ wo er den kosmopolitischen Geist des Banus hervorhebt und lobt. Er sei der Banus aller Kroaten, die immer sehr liberale Petitionen geschrieben hätten.

Aus den erwähnten Beiträgen im Sloveniens Blatt geht deutlich hervor, dass die Zeitung dem Banus Jelačić trotz seinem Verhalten in den Oktoberereignissen zugeneigt war. Das zeigte sich auch später, z.B. bei einer im November herausgebrachten Anekdote über ihn, die aus der österreichischen Zeitung Volksblatt übernommen worden war. Eine Anecdote von Jellachich ${ }^{56}$ brachte dem Lesepublikum menschliche Eigenschaften des Banus nahe, vor allem seine Wohltätigkeit und Sinnlichkeit. Dem Text nach habe er die Soldaten vor grimmiger Kälte gerettet, indem er ihnen auf eigenes Risiko frühzeitig den Abmarschbefehl gegeben habe. Das sei „im Februar heurigen Jahres“ passiert, als Jelačić noch Oberst war. Seine Truppen seien bereit zum Abmarsch von Carlstadt nach Italien gewesen und hätten in der Kälte schon zwei Stunden auf den Befehl gewartet. Nach dem Ereignis wollte der General, dass Jelačić „,cassiirt“ werde, denn schließlich ging es ja um „ein schweres Subordinations-Vergehen“. Der Wiener Hofkriegsrat wollte dagegen Jelačić wegen seiner Verdienste nur „pensionieren“. Aber dann kam die Revolution „und Jelachich wurde Ban“. Die Revolution sei für ihn also eine Rettung gewesen. ${ }^{57}$ Die in der Kälte stehenden Soldaten warteten auf den Abmarschbefehl des k. k. Generalfeldzeugmeisters Franz Freiherren Dahlen von Orlaburg, ${ }^{58}$ des ehemaligen Vorgesetzten des Oberst Jelačić und des Präsidenten des obersten Militärgerichtshofs. Also es ging um eine hochpositionierte Person des damaligen militärisch-politischen Lebens, dessen Befehle schon von Anfang an im Gegensatz zu denen von Jelačić standen. Sie waren oft in Diskrepanz zu dem, was das Innenministerium verlangte bzw. befahl und Jelačić deshalb nicht erfüllen konnte. Kein Wunder also, dass der dem Kaiser treue Banus sich oft über den General aufregte. ${ }^{59}$

Es ist interessant, dass die Stimmung der erwähnten Anekdote näher der Meinung des jungen Hermann Dahlen ${ }^{60}$ war. Der Sohn des berühmten Generals wurde vom ersten Tage dem Hauptquartier zugeteilt und dadurch in die Lage versetzt, aus unmittelbarer Nähe das ganze Geschehen um den Banus zu beobachten und Jelačić

55 Einfluß des südslavischen Krieges auf den österreichischen Gesammtstaat, Sloveniens Blatt, 10. 10. 1848, 57-58, und 17. 10. 1848, 61-62.

56 Eine Anecdote von Jellachich, Sloveniens Blatt, 28. 11. 1848, 87.

57 Sloveniens Blatt, 28. 11. 1848, 87.

58 Franz Dahlen von Orlaburg (6. 12. 1779, Orlat, Siebenbürgen - † 18. 2. 1859 Graz). Dieses Geschlecht stammt aus Rußland, er wurde österreichischer Ritter 18. 5. 1840. Peter FrankDöfering, Adelslexikon des österreichischen Kaisertums 1804-1918 (Wien, Freiburg, Basel: Herder Verlag, 1989), 272.

59 Josip Neustädter, Ban Jelačić i događaji u Hrvatskoj, od godine 1848, Band 1, 327.

60 Hermann Josua Anton Freiherr Dahlen von Orlaburg (10. 1. 1828, Kaschau - †13. 11. 1887 Wien), www.biographien.ac.at (Zugriff: 6. 7. 2019). Feldmarschall-Leutnant und Commandant der 9. Infanteriedivision zu Prag. Gothaisches genealogisches Taschenbuch der freiherrlichen Häuser (Gotha: Verlag Justus Perthes, 1876), 33. 
mitzuerleben. ${ }^{61}$ In seinem Tagebuch beschrieb er unter anderem die Belagerung Wiens im Oktober 1848. Er bezeichnete zwar Jelačić als Löwe, meinte aber, der würde trotz allem sehr lange ruhen. ${ }^{62}$ Über die Einheit und Homogenität unter den Soldaten, für die der Banus sorgte, schrieb er in seinem Tagebuch folgendes: „Es ist wirklich als wären wir alle Mitglieder einer Familie unter einem vielgeliebten Vater, der alle Herzen an sich kettet und dadurch Alle untereinander wieder in Liebe verbindet. ${ }^{\text {" }} 3$ Jelačić blieb also in seinen Erinnerungen im sehr positiven Lichte, also als eine starke Person, obwohl er schon vor der Revolution gesundheitliche Probleme hatte. ${ }^{64}$

Anhand der veröffentlichten Nachrichten und Artikel in Sloveniens Blatt kann festgestellt werden, dass die Zeitung dem Banus zugeneigt war, was sie auch offen und nicht zurückhaltend ausdrückte. Im Unterschied zu der Cillier Zeitung blieb diese Zuneigung im Sloveniens Blatt auch nach den Oktoberereignissen eindeutig.

\section{Fazit}

In den Zeitungen wiederspiegeln sich die Zeiten, in denen sie herausgebracht werden, und weil das Jahr 1848 ein schicksalhaftes Jahr war, tragen besonders durch die Revolution "geborenen“ Zeitungen einen ausgeprägt politischen Charakter. Das gilt auch für die Zeitungen, die in slowenisch besiedelten Gebieten im Jahre 1848 herausgebracht wurden. Dabei traten die Kreisstädte Novo Mesto und Celje besonders hervor. Aus diesen Städten stammen die ,jungen“ deutschsprachigen Zeitungen, deren Redakteure liberal orientiert waren und die das Lesepublikum möglichst ausführlich über die aktuellen Ereignisse und Themen (objektiv) informieren wollten. Die gedruckten Mitteilungen und Artikel bieten dem gegenwärtigen Leser und Forscher zwischen den Zeilen noch weitere Informationen an. Beim aufmerksamen Lesen können versteckte Meinungen des Redakteurs oder Ideen eines Autors entdeckt werden. Im vorliegenden Beitrag wurde anhand der ,jungen“, durch die Revolution herausgebrachten Zeitungen aus den erwähnten Städten - die Cillier Zeitung und das Sloveniens Blatt - ein Versuch unternommen, die Beziehung slowenischer, liberal orientierter politischer Öffentlichkeit zu Jelačić festzustellen. Die anderen „jungen“ deutschsprachigen Zeitungen im damaligen slowenischen Raum wurden in die Recherche nicht einbezogen, weil sie keine Berichte über ihn enthalten.

Nach den Erwartungen waren die beiden erwähnten Zeitungen begeistert mit den Ergebnissen der Revolution und folglich auch mit den wichtigsten (politischen) Protagonisten dieser Zeit, so auch mit Josip Jelačić. In den herausgebrachten Artikeln und Notizen spürt man besonders am Anfang vor allem Begeisterung und Zuneigung. Da das Sloveniens Blatt national stärker geprägt war, war die Zuneigung vielleicht

$61 \quad$ Ferdinand Hauptmann, Jelačićs Kriegszeug nach Ungarn 1848 (Graz: Historisches Institut der Universität, Abt. Südosteuropäische Geschichte, 1975), 4.

62 Hauptmann, Jelačićs Kriegszeug nach Ungarn 1848, 9.

63 Hauptmann, Jelačićs' Kriegszeug nach Ungarn 1848, 11.

64 Walter Görlitz, Jelačić. Symbol für Kroatien. Die Biographie (Wien; München: Amalthea Signum 1992), 30-33; Josip Neustädter, Ban Jelačić i događaji u Hrvaskoj, od godine 1848, Band 2, 306-336. 
sogar größer, auf alle Fälle aber offensichtlicher. Das Blatt drückte seine Begeisterung stärker und ersichtlicher aus, wobei die extrem liberale Cillier Zeitung dabei zurückhaltend blieb. Das Sloveniens Blatt brachte auch längere Artikel über den Banus, während in der Cillier Zeitung das selten vorkam. Da wurden über ihn oft nur kurze Notizen gebracht, was noch deutlicher nach dem Oktober 1848 wurde. Bei den Oktoberereignissen blieben jedoch beide Zeitungen zurückhaltend obwohl nicht kritisch bezüglich Jelačićs Aktivitäten in den Kämpfen. Vielmehr könnte man zwischen den Zeilen gewisse Rechtfertigung herauslesen.

Nach dieser Revolutionsphase gingen die untersuchten Zeitungen andere und auch unterschiedliche Wege. In der Cillier Zeitung erschienen nur noch sachliche, oft nachgedruckte Artikel über den Banus. Das Sloveniens Blatt dagegen berichtete über den Banus weiterhin mit offener Begeisterung. 


\section{Literatur- und Quellenverzeichnis}

Ambrožič, Matjaž. Ljubljanski knezoškof dr. Janez Zlatoust Pogačar. Ljubljana: Acta Ecclesiastica Sloveniae, 2000.

Cillier Zeitung (1848).

Gatti, Ferdinand. Die Ereignisse des Jahres 1848 in der Steiermark. Graz: In Commision bei J. Kienrich, 1850.

Frank-Döfering, Peter. Adelslexikon des österreichischen Kaisertums 1804-1918. Wien, Freiburg, Basel: Herder Verlag, 1989.

Görlitz, Walter. Jelačić. Symbol für Kroatien. Die Biographie. Wien; München: Amalthea Signum, 1992.

Gothaisches genealogisches Taschenbuch der freiherrlichen Häuser. 26. Jhrg. Gotha: Verlag Justus Perthes, 1876.

Granda, Stane. „Sloveniens Blatt - časopis iz leta 1848“. Zgodovinski časopis = Historical review: glasilo Zveze zgodovinskih društev Slovenije 48 (1994), Nr. 2: 231-252.

Granda, Stane. Dolenjska v revolucionarnem letu 1848. Novo Mesto: Dolenjska založba, Znanstveno-raziskovalni center, 1995.

Granda, Stane. Prva odločitev Slovencev za Slovenijo. Ljubljana: Korenine, 1999.

Hauptmann, Ferdinand. Jelačić's Kriegszeug nach Ungarn 1848. Graz: Historisches Institut der Universität, Abt. Südosteuropäische Geschichte, 1975.

Horvat, Rudolf. Ban Jelačić. Prvi svezak, Hrvatski pokret 1848. Zagreb: Colorprint Zagreb, 1990.

Hrvatski biografski leksikon, online http://hbl.lzmk.hr

Ilešič, Fran. O slovensko-hrvatski zajednici 1848/49, Bleiweisov zbornik, 278-319. Ljubljana: Matica Slovenska, 1909.

Jakopec, Miloš. „Časnikarstvo v Novem mestu: pregled od prvih začetkov do II. svetovne vojne“. Rast (1990), Nr. 2: 110-119.

Laibacher Kirchenzeitung (1848).

Laibacher Zeitung (1848).

Korunić, Petar. Jugoslavenska ideologija u hrvatskoj i slovenskoj politici: Hrvatsko-slovenski politički odnosi 1848-1870 (Zagreb: Globus: Centar za povijesne znanosti sveučilišta u Zagrebu, Odjel za hrvatsku povijest, 1986).

Meynert, Hermann. Geschichte der Ereignisse in der österreichischen Monarchie während der Jahre 1848 u. 1849 in ihren Ursachen und Folgen (Zugleich als Suppl. zu „Gesch. Oesterreichs“ vom Verfasser). Wien: Verlag und Druck von Carl Gerold und Sohn, 1853.

Miladinović Zalaznik, Mira; Granda, Stane, Hg. Anton Aleksander Graf AuerspergAnastazij Grün. Razprave [Anton Alexander Graf Auersperg - Anastasius Grün. Abhandlungen]. Ljubljana: Nova revija, 2009.

Neustädter, Josip. Ban Jelačić i događaji u Hrvatskoj, od godine 1848. Zagreb: Školska knjiga, 1998. 
Milković, Kristina. Josip Jelačić 1830.-1848. Plemić, časnik i preporoditelj u društvima „staroga režima”. Magisterarbeit, Sveučilište u Zagrebu, 2009.

Österreichische Biographische Lexikon, online https://www.biographien.ac.at

Pejaković, Stephan. Aktenstücke zur Geschichte des kroatisch-slawonischen Landtages und der nationalen Bewegung vom Jahre 1848: mit einem Anhange, enthaltend: Die wichtigsten Landtags-Akten vom Jahre 1861, und das Programm von O. Ostrožinski aus dem Jahre 1848: Nebst Porträt des Ban Jellačić. Wien: Mechitharisten-Buchdruckerei, 1861.

Peternel, Marija Mojca. „Slovenci v luči augsburškega Allgemeine Zeitung v revolucionarnem letu 1848/1849“, Časopis za zgodovino in narodopisje 87 (2016), Nr. 1-2: 21-41.

Peternel, Marija Mojca. „Die Laibacher Kirchenzeitung: Pressefreiheit und Kirche im Revolutionsjahr 1848“. Blätter für Heimatkunde 89 (2015), Heft 1-2: 25-41.

Peternel, Marija Mojca. Cillier Wochenblatt (Zeitung): celjski nemški časopis iz leta 1848. Celje: Zgodovinsko društvo, 2006.

Pogoršek, Nataša. Slovenska hrvaška vzajemnost. Maribor: Zgodovinsko društvo dr. Franca Kovačiča v Mariboru, 2009.

Rahten, Andrej. Zavezništva in delitve. Razvoj slovensko-hrvaških političnih odnosovv Habsburški monarhiji. Ljubljana: Nova revija, 2005.

Sloveniens Blatt (1848).

Slovenska biografija, online https://www.slovenska-biografija.si

Stančić, Nikša. „JELAČIĆ, Josip (Jellačić, Jellachich; Joseph)“. U: Hrvatski biografski leksikon, Band 6, 394-401. Zagreb: Leksikografski zavod Miroslav Krleža, 2005.

Šidak, Jaroslav. Studije iz hrvatske povijesti za revolucije 1848-49. Zagreb: Centar za povijesne znanosti Sveučilišta, Odjel za hrvatsku povijest, 1979. 
Marija Mojca Peternel

\section{Slovenske novine na njemačkom jeziku i ban Jelačić u revolucionarnoj 1848. godini \\ Analiza na primjeru novina Cillier Zeitung i Sloveniens Blatt}

\section{Sažetak}

Kao rezultat ukidanja cenzure u ožujku 1848. godine širom Habsburške Monarhije pojavljuju se nove novine, a sloboda tiska imala je konkretnog odjeka i u zemljama sa slovenskim stanovništvom. U ovo istraživanje uključene su samo novine na njemačkom jeziku koje je "iznjedrila” revolucija. Njihov je jezik bio njemački jer je u to vrijeme taj jezik bio daleko poznatiji slovenskim obrazovanim građanima nego slovenski. Budući da je godinu 1848./49. obilježila revolucija, a novine su uvijek zrcalo svoga vremena, i ovi su listovi imali izražen politički karakter. U tom smo okviru željeli ispitati njihov stav prema banu Josipu Jelačiću i način na koji su reagirali na njegove aktivnosti tijekom revolucionarne godine.

Ključne riječi: sloboda tiska, revolucija 1848./49., Josip Jelačić, slovenske novine na njemačkom jeziku

Marija Mojca Peternel, Oddelek za germanistiko z nederlandistiko in skandinavistiko Ljubljana, Filozofska fakulteta Univerza v Ljubljani, Aškerčeva 2, 1000 Ljubljana, Republika Slovenija, E-mail adresa: mojca.peternel@ff.uni-lj.si 